\title{
Derivations of some filiform Leibniz algebras
}

\author{
AL-hossain Ahmad, Khiyar AL-hossain \\ Department of Mathematics, AL Qunfudha University College, Umm AL Qura University, City of AL Qunfudha, Kingdom of Saudi Arabia
}

Email address:

hossa-1427@hotmail.com (AL-hossain A.), asas.1429@hotmail.com (K. AL-hossain)

\section{To cite this article:}

AL-hossain Ahmad, Khiyar AL-hossain. Derivations of Some Filiform Leibniz Algebras. Pure and Applied Mathematics Journal. Vol. 3, No. 6, 2014, pp. 121-125. doi: 10.11648/j.pamj.20140306.12

\begin{abstract}
In this paper the two classes of filiform Leibniz algebras $\mu_{0}^{n}$ and $\mu_{1}^{n}$ in $(n+1)$ dimensions of filiform Leibniz algebras such that $n \geq 2$ will be considered. The study includes derivations of naturally graded Leibniz algebras of first class $L_{n}$ and second class $W_{n}$, be algebras whose multiplications rules are defined by the $\mu_{0}^{n}$ and $\mu_{1}^{n}$, respectively. The algebras of derivations of naturally graded Leibniz algebras are described by linear transformations and dimensions derivations. Finally, we determine number of derivations of naturally graded Leibniz algebras.
\end{abstract}

Keywords: Leibniz Algebra, Filiform Leibniz Algebra, Characteristically Nilpotent Algebra, Graded Leibniz Algebra, Derivation

\section{Introduction}

In 1955 Jacobson established that, over a field of characteristic zero, any Lie algebra which has nondegenerate derivations is nilpotent [17]. In the same paper [11] he asked for the converse. This result is assumed to be the origin of the theory of characteristically nilpotent Lie algebras. An example of a nilpotent Lie algebra all of whose derivations are nilpotent (hence degenerate), answering the above question negatively, was constructed in Dixmier and Lister [6]. Lie algebras whose derivations are nilpotent endomorphisms have been called characteristically nilpotent. The result of Dixmier and Lister is assumed to be the origin of the theory of characteristically nilpotent Lie algebras. They defined a generalization of the central descending sequence and called the algebras satisfying the nullity of a power characteristically nilpotent.The (co)homology theory, representations and related problems of Leibniz algebras were studied by Loday. and Pirashvili, [15], and others.Since the class of Leibniz algebras are a noncommutative generalization of the class of Lie algebras, we naturally face the problem of finding a good relationship (as well as in the case of Lie algebras [7]) for the algebra of derivations of L. The investigations in the present paper are devoted to this problem. In particular, for naturally graded complex Leibniz algebras, we describe their algebras of derivations generating the algebras of derivations of filiform Leibniz algebras. This description enables us to distinguish the characteristically nilpotent algebras in the class of filiform Leibniz algebras. In the present paper we study the derivation algebras of lowdimensional Leibniz algebras. The outline of the paper is as follows. Section 1 is a brief introduction. Section 2 we give derivations of filiform Leibniz algebras $L_{n}$. In Section 3 we give derivations of filiform Leibniz algebras $W_{n}$.

Definition 1.1. [17] An algebra L over F a field is said to be Leibniz if the Leibniz identity:

$$
[\mathrm{x},[\mathrm{y}, \mathrm{z}]]=[[\mathrm{x}, \mathrm{y}], \mathrm{z}]+[[\mathrm{x}, \mathrm{z}], \mathrm{y}]
$$

holds for any $\mathrm{x}, \mathrm{y}, \mathrm{z}$ in $\mathrm{L}$, where [,] stands for the multiplication in L.

Note that if the identity $[\mathrm{x}, \mathrm{x}]=0$ holds in $\mathrm{L}$, then the Leibniz identity becomes the Jacobi identity. Thus, the Leibniz algebras are the "noncommutative" analog of Lie algebras. For an arbitrary algebra L, we define the sequence

$$
L^{1} \equiv L \quad L^{n+1}=\left[L^{n}, L\right] .
$$

Definition 1.2. [18] A Leibniz algebra $\mathrm{L}$ is said to be nilpotent if there exists a positive integer $\mathrm{s}$ in $\mathrm{N}$ such that $L^{1} \supset L^{2} \supset \ldots \supset L^{s}=0$ The smallest integer s for which $L^{\mathrm{s}}=0$ is called the nilindex of $\mathrm{L}$.

Definition 1.3. [7] An n-dimensional Leibniz algebra L is said to be filiform if $\operatorname{dim} \mathrm{L}^{\mathrm{i}}=\mathrm{n}-\mathrm{i}$, where $2 \leq i \leq n$.

Definition 1.4. [1] Given a filiform Leibniz algebra L, put $L_{i}=L^{i} / L^{i+1}, 1 \leq \mathrm{i} \leq$ and $g r L=L_{1} \ominus L_{2} \ominus \ldots \ominus L_{n-1}$. Then $\left[L_{i}, L_{j}\right] \subseteq L_{i+j}$ and we obtain the graded algebra grL. If grL and $\mathrm{L}$ are isomorphic, denoted by $\mathrm{grL}=\mathrm{L}$, we say that the algebra $\mathrm{L}$ is naturally graded. 
Theorem 1.1. [2] Every $(\mathrm{n}+1)$-dimensional naturally graded complex non-Lie filiform Leibniz algebra is isomorphic to one of the following two algebras:

$$
\begin{aligned}
& \mu_{0}^{n}:\left[\mathrm{e}_{0}, \mathrm{e}_{0}\right]=\mathrm{e}_{2},\left[\mathrm{e}_{\mathrm{i}}, \mathrm{e}_{0}\right]=\mathrm{e}_{\mathrm{i}}+1,1 \leq i \leq \mathrm{n}-1 \\
& \mu_{1}^{n}:\left[\mathrm{e}_{0}, \mathrm{e}_{0}\right]=\mathrm{e}_{2},\left[\mathrm{e}_{\mathrm{i}}, \mathrm{e}_{0}\right]=\mathrm{e}_{\mathrm{i}}+1,2 \leq i \leq \mathrm{n}-1
\end{aligned}
$$

where $\mathrm{e}_{0}, \mathrm{e}_{1}, \ldots, \mathrm{e}_{\mathrm{n}}$ is a basis of the algebra $\mathrm{L}$.

Corollary 1.1. [2] Every ( $n+1)$-dimensional complex nonLie filiform Leibniz algebra is isomorphic to one of the following non-Lie filiform Leibniz algebras:

$$
\begin{gathered}
\mu_{0}^{n}(\beta):\left[\mathrm{e}_{0}, \mathrm{e}_{0}\right]=\mathrm{e}_{2},\left[\mathrm{e}_{\mathrm{i}}, \mathrm{e}_{0}\right]=\mathrm{e}_{\mathrm{i}+1}, 1 \leq i \leq n-1, \\
{\left[\mathrm{e}_{0}, \mathrm{e}_{1}\right]=\alpha_{3} \mathrm{e}_{3}+\alpha_{4} \mathrm{e}_{4}+\ldots+\alpha_{\mathrm{n}-1} \mathrm{e}_{\mathrm{n}-1}+\theta_{\mathrm{en}}} \\
{\left[\mathrm{e}_{\mathrm{i}}, \mathrm{e}_{1}\right]=\alpha_{3} \mathrm{e}_{\mathrm{i}+2}+\alpha_{4} \mathrm{e}_{\mathrm{i}+3}+\ldots+\alpha_{\mathrm{n}+1-\mathrm{i}} \mathrm{e}_{\mathrm{n}}, 1 \leq i \leq n-2}
\end{gathered}
$$

where $\alpha_{3}, \alpha_{4}, \ldots, \alpha_{n}, \theta \in C$, and

$\mu_{1}^{n}(\beta):\left[\mathrm{e}_{0}, \mathrm{e}_{0}\right]=\mathrm{e}_{2},\left[\mathrm{e}_{\mathrm{i}}, \mathrm{e}_{0}\right]=\mathrm{e}_{\mathrm{i}+1}, 2 \leq i \leq n-1$,

$$
\begin{gathered}
{\left[\mathrm{e}_{0}, \mathrm{e}_{1}\right]=\beta_{3} \mathrm{e}_{3}+\beta_{4} \mathrm{e}_{4}+\ldots+\beta_{\mathrm{n}} \mathrm{e}_{\mathrm{n}},\left[\mathrm{e}_{1}, \mathrm{e}_{1}\right]=\gamma \mathrm{e}_{\mathrm{n}},} \\
{\left[\mathrm{e}_{\mathrm{i}}, \mathrm{e}_{1}\right]=\beta_{3} \mathrm{e}_{\mathrm{i}+2}+\beta_{4} \mathrm{e}_{\mathrm{i}+3}+\ldots+\beta_{\mathrm{n}+1}-\mathrm{e}_{\mathrm{n}}, 2 \leq i \leq n-2,}
\end{gathered}
$$

where $\beta_{3}, \beta_{4}, \ldots, \beta_{\mathrm{n}}, \gamma \in \mathrm{C}$, and $\mathrm{e}_{0}, \mathrm{e}_{1}, \ldots, \mathrm{e}_{\mathrm{n}}$ is a basis of the algebra L.

Definition 1.5. [1] A linear transformation d of a Leibniz algebra $\mathrm{L}$ is called a derivation if for any $\mathrm{x}, \mathrm{y}$ in $\mathrm{L}$

$$
\mathrm{d}([\mathrm{x}, \mathrm{y}])=[\mathrm{d}(\mathrm{x}), \mathrm{y}]+[\mathrm{x}, \mathrm{d}(\mathrm{y})]
$$

The space of all derivations of the algebra L equipped with the multiplication defined as the commutator, forms a lie algebra which is denoted by $\operatorname{Der}(\mathrm{L})$. It is clear that the operator of right multiplication $\mathrm{R}_{\mathrm{x}}$ by an element $\mathrm{x}$ of the algebra $\mathrm{L}$ (that is $\mathrm{R}_{\mathrm{x}}(\mathrm{y})=[\mathrm{y}, \mathrm{x}]$ ) is also derivation. Derivations of this type are called inner derivations. Similar to the lie algebras case the set of the inner derivation $\operatorname{Inn}(\mathrm{L})$ forms an ideal of the algebra $\operatorname{Der}(\mathrm{L})$.

Lemma 1.1. [17] For any $r \leq(p-1)(1-j)$, , one has $F_{r} Z^{j}$ $(L, L)=Z^{\mathrm{j}}(L, L)$.

Let $\mathrm{L}_{\mathrm{n}}$ and $\mathrm{W}_{\mathrm{n}}$ be algebras whose multiplication rules are defined by the multiplication $\mu_{0}^{n}$ and $\mu_{1}^{n}$, respectively.

\section{Derivation of Filiform Leibniz Algebra $L_{n}$}

Proposition 2.1. The linear transformations $\mathrm{t}_{1}, \mathrm{t}_{2}, \mathrm{t}_{3}, \mathrm{t}_{4}$ and $\mathrm{d} \mathrm{k}, 1 \leq \mathrm{k} \leq \mathrm{n}-2$ of $\mathrm{L}_{\mathrm{n}}$ defined by the rules:

$$
\begin{aligned}
& \mathrm{t}_{1}\left(\mathrm{e}_{0}\right)=\mathrm{e}_{0}, \mathrm{t}_{1}\left(\mathrm{e}_{\mathrm{i}}\right)={ }_{\mathrm{i}} \mathrm{e}_{\mathrm{i}}, 1 \leq \mathrm{i} \leq \mathrm{n}, \\
& \mathrm{t}_{2}\left(\mathrm{e}_{0}\right)=\mathrm{e}_{1}, \mathrm{t}_{2}\left(\mathrm{e}_{\mathrm{i}}\right)=\mathrm{e}_{\mathrm{i}}, 1 \leq \mathrm{i} \leq \mathrm{n}, \\
& \mathrm{t}_{3}\left(\mathrm{e}_{0}\right)=\mathrm{e}_{\mathrm{n}} \\
& \mathrm{t}_{4}\left(\mathrm{e}_{1}\right)=\mathrm{e}_{\mathrm{n}} \\
& \mathrm{d}_{\mathrm{k}}\left(\mathrm{e}_{0}\right)=\mathrm{e}_{\mathrm{k}+1}, \mathrm{~d}_{\mathrm{k}}\left(\mathrm{e}_{\mathrm{i}}\right)=\mathrm{e}_{\mathrm{k}+\mathrm{i}}, 1 \leq \mathrm{i} \leq \mathrm{n}-\mathrm{k} .
\end{aligned}
$$

form a basis of the space $\operatorname{Der}\left(\mathrm{L}_{\mathrm{n}}\right)$.

Proof. We introduce a grading of the algebra by setting $L_{n}$ $=L_{1} \oplus L_{2} \oplus \ldots \oplus L_{i}$ by setting $L_{1}=\operatorname{lin}\left(e_{0}, e_{1}\right), L_{i}=\operatorname{lin}\left(e_{i}\right)$ for $2 \leq i \leq n$. Since $Z_{l}\left(L_{n}, L_{n}\right)=\operatorname{Der}\left(L_{n}\right)$ and grading of the algebra $L_{n}$ is finite, there is a finite grading in the space $\operatorname{der}\left(L_{n}\right)$. Let $\mathrm{d}$ in $\operatorname{Der}\left(L_{\mathrm{n}}\right)$. In this case, by Lemma 1.1 we have $\mathrm{d}=\mathrm{d}_{0}+\mathrm{d}_{1}+\ldots+\mathrm{d}_{\mathrm{n}-2}+\mathrm{d}_{\mathrm{n}-1}$, where $d_{\mathrm{i}}$ in $\operatorname{Der}\left(L_{\mathrm{n}}\right)$ and $\mathrm{d}_{\mathrm{i}}\left(\mathrm{L}_{\mathrm{j}}\right) \subseteq$ $\mathrm{L}_{\mathrm{i}+\mathrm{j}}$.

Consider the element $\mathrm{d}_{0} \in \operatorname{Der}\left(\mathrm{L}_{\mathrm{n}}\right)$ It is clear that

$$
\mathrm{d}_{0}\left(\mathrm{e}_{i}\right)=\left\{\begin{array}{c}
\alpha_{\mathrm{o}} \mathrm{e}_{\mathrm{o}}+\alpha_{1} \mathrm{e}_{1}, \text { for } i=0, \\
\beta_{0}+\beta_{1} \beta_{1}, \text { for } i=1, \\
\gamma_{i} \mathrm{e}_{i}, \text { for } 2 \leq i \leq \mathrm{n},
\end{array}\right.
$$

where $\alpha_{0}, \alpha_{1}, \beta_{0}, \beta_{1}, \gamma_{i}, 2 \leq i \leq \mathrm{n}$, are scalars (elements of the field).

Consider the family of derivations

$$
\mathrm{d}_{0}\left(\left[\mathrm{e}_{i}, \mathrm{e}_{j}\right]\right)=\left[\mathrm{d}_{0}\left(\mathrm{e}_{\mathrm{i}}\right), \mathrm{e}_{\mathrm{j}}\right]+\left[\mathrm{e}_{\mathrm{i}}, \mathrm{d}_{0}\left(\mathrm{e}_{j}\right)\right] .
$$

If $j=1$ we obtain

$$
\left[\mathrm{e}_{i}, \mathrm{~d}_{0}\left(\mathrm{e}_{1}\right)\right]=\left[\mathrm{e}_{i}, \beta_{0} \mathrm{e}_{0}+\beta_{1} \mathrm{e}_{1}\right]=\beta_{0} \mathrm{e}_{\mathrm{i}+1}=0 \Rightarrow \beta_{0}=0
$$

If $i=1$ and $j=0$ then

$$
\begin{gathered}
\mathrm{d}_{0}\left(\mathrm{e}_{2}\right)=\left[\mathrm{d}_{0}\left(\mathrm{e}_{1}\right), \mathrm{e}_{0}\right]+\left[\mathrm{e}_{1}, \mathrm{~d}_{0}\left(\mathrm{e}_{0}\right)\right] \Rightarrow \gamma_{2} \mathrm{e}_{2}=\left(\beta_{1}+\alpha_{0}\right) \mathrm{e}_{2} \Rightarrow \\
\gamma_{2}=\beta_{1}+\alpha_{0}
\end{gathered}
$$

i.e., $\gamma_{2}=\beta_{1}+\alpha_{0}$

If $\mathrm{i}=0$ and $j=0$ then

$\mathrm{d}_{0}\left(\mathrm{e}_{2}\right)=\left[\mathrm{d}_{0}\left(\mathrm{e}_{0}\right), \mathrm{e}_{0}\right]+\left[\mathrm{e}_{0}, \mathrm{~d}_{0}\left(\mathrm{e}_{0}\right)\right] \Rightarrow \gamma_{2} \mathrm{e}_{2}=\alpha_{0} \mathrm{e}_{2}+\alpha_{1} \mathrm{e}_{2}+$ $\alpha_{0} \mathrm{e}_{2} \Rightarrow \gamma_{2} \mathrm{e}_{2}=\left(2 \alpha_{0}+\alpha_{1}\right) \mathrm{e}_{2}$

i.e, $\gamma_{2}=2 \alpha_{0}+\alpha_{1}$

If $\mathrm{j}=0$ and $2 \leq i \leq n$ we obtain

$$
\mathrm{d}_{0}\left(\mathrm{e}_{\mathrm{i}+1}\right)=\gamma_{\mathrm{i}} \mathrm{e}_{\mathrm{i}+1}+\alpha_{0} \mathrm{e}_{\mathrm{i}+1} \Rightarrow \gamma_{\mathrm{i}+1}=\gamma_{\mathrm{i}}+\alpha_{0}
$$

However, since $\gamma_{2}=2 \alpha_{0}+\alpha_{1}$ it follows that $\gamma_{1}=i \alpha_{0}+\alpha$ Thus,

$\mathrm{d}_{0}\left(\Sigma_{i}^{n}={ }_{0} \lambda_{\mathrm{i}} \mathrm{e}_{\mathrm{i}}\right)=\lambda_{0}\left(\alpha_{0}+\alpha_{1} \mathrm{e}_{1}\right)+\lambda_{1}\left(\alpha_{0} \mathrm{e}_{1}+\alpha_{1} \mathrm{e}_{1}\right)+\Sigma_{i}^{n}={ }_{2} \lambda_{\mathrm{i}}$ $\mathrm{e}_{\mathrm{i}}\left(\mathrm{i}_{\mathrm{i}} \alpha_{0}+\alpha_{1}\right)=\alpha_{0}\left(\lambda_{0} \mathrm{e}_{0}+\sum_{i=1}^{n} i \lambda_{\mathrm{i}} \mathrm{e}_{\mathrm{i}}\right)+\alpha_{1}\left(\lambda_{0} \mathrm{e}_{1}+\sum_{i=1}^{n} \lambda_{\mathrm{i}} \mathrm{e}_{\mathrm{i}}\right)$, i.e., $d_{0}=\alpha_{0} t_{1}+\alpha_{1} t_{2}$

Consider the elements $d_{\mathrm{k}} \in \operatorname{Der}\left(\mathrm{L}_{\mathrm{n}}\right)$ for $1 \leq k \leq n-2$.It is clear that

$$
d_{\mathrm{k}}\left(\mathrm{e}_{i}\right)=\left\{\begin{array}{c}
\tau_{o} \mathrm{e}_{\mathrm{k}+1}, \quad \text { for } i=0, \\
\tau_{i} \mathrm{e}_{\mathrm{k}+\mathrm{i}}, \quad \text { for } 1 \leq \mathrm{i} \leq n-\mathrm{k},
\end{array}\right.
$$

where $\tau_{i}, 0 \leq i \leq n-k$ are scalars (elements of the field).

Consider the following property of the derivations

$$
\mathrm{d}_{\mathrm{k}}\left(\left[\mathrm{e}_{\mathrm{i}}, \mathrm{e}_{\mathrm{j}}\right]\right)=\left[\mathrm{d}_{\mathrm{k}}\left(\mathrm{e}_{\mathrm{i}}\right), \mathrm{e}_{\mathrm{j}}\right]+\left[\mathrm{e}_{\mathrm{i}}, \mathrm{d}_{\mathrm{k}}\left(\mathrm{e}_{\mathrm{j}}\right)\right] .
$$

If $\mathrm{j}=0$ and $\mathrm{i}=1$, we have

$$
\mathrm{d}_{\mathrm{k}}\left(\mathrm{e}_{2}\right)=\tau_{1}\left[\mathrm{e}_{\mathrm{k}+1}, \mathrm{e}_{0}\right] \Rightarrow \tau_{2} \mathrm{e}_{\mathrm{k}+2}=\tau_{1} \mathrm{e}_{\mathrm{k}+2} \Rightarrow \tau_{2}=\tau_{1} .
$$

If $\mathrm{j}=0$ and $\mathrm{i}=0$, we have

$$
\mathrm{d}_{\mathrm{k}}\left(\mathrm{e}_{2}\right)=\tau_{0}\left[\mathrm{e}_{\mathrm{k}+1}, \mathrm{e}_{0}\right] \Rightarrow \tau_{2} \mathrm{e}_{\mathrm{k}+2}=\tau_{0} \mathrm{e}_{\mathrm{k}+2} \Rightarrow \tau_{2}=\tau_{0} .
$$


if $\mathrm{j}=0$ and $1 \leq \mathrm{i} \leq n-\mathrm{k}$,we obtain

$$
\mathrm{d}_{\mathrm{k}}\left(\mathrm{e}_{\mathrm{i}+1}\right)=\tau_{i} \mathrm{e}_{\mathrm{k}+\mathrm{i}+1} \Rightarrow \tau_{\mathrm{i}+1} \mathrm{e}_{\mathrm{k}+\mathrm{i}+1}=\tau_{\mathrm{i}} \mathrm{e}_{\mathrm{k}+\mathrm{i}+1} \Rightarrow \tau_{\mathrm{i}+1}=\tau_{i} .
$$

i.e., $\tau_{0}=\tau_{1}=\ldots=\tau_{\mathrm{n}-\mathrm{k}}$.

Thus,

$$
\mathrm{d}_{\mathrm{k}}\left(\Sigma_{i=0}^{n} \lambda_{\mathrm{i}} \mathrm{e}_{\mathrm{i}}\right)=\tau_{0}\left(\lambda_{0} \mathrm{e}_{\mathrm{k}+1}+\Sigma_{i=1}^{n-1} \lambda_{\mathrm{i}} \mathrm{e}_{\mathrm{k}+\mathrm{i}}\right) .
$$

Consider the elements $d_{n-1} \in \operatorname{Der}\left(L_{n}\right)$. It is clear that

$$
\mathrm{d}_{\mathrm{n}-1}\left(\mathrm{e}_{i}\right)= \begin{cases}\delta_{\mathrm{o}} e_{n}, & \text { for } \mathrm{i}=0, \\ \delta_{1} \mathrm{e}_{\mathrm{n}}, & \text { for } \mathrm{i}=1,\end{cases}
$$

where $\delta_{0}$, and $\delta_{1}$ are scalars (elements of the field).

Consider the following property of the derivations

$$
\mathrm{d}_{\mathrm{n}-1}\left(\left[\mathrm{e}_{\mathrm{i}}, \mathrm{e}_{\mathrm{j}}\right]\right)=\left[\mathrm{d}_{\mathrm{n}-1}\left(\mathrm{e}_{i}\right), \mathrm{e}_{\mathrm{j}}\right]+\left[\mathrm{e}_{\mathrm{i}}, \mathrm{d}_{\mathrm{n}-1}\left(\mathrm{e}_{\mathrm{j}}\right)\right] .
$$

If $\mathrm{i}=0$ and $\mathrm{j}=0$, we have

$$
\mathrm{d}_{\mathrm{n}-1}\left(\mathrm{e}_{2}\right)=\delta_{0}\left[\mathrm{e}_{\mathrm{n}}, \mathrm{e}_{0}\right]+\delta_{0}\left[\mathrm{e}_{0}, \mathrm{e}_{\mathrm{n}}\right] \Rightarrow 0=0
$$

If $\mathrm{i}=1$ and $\mathrm{j}=0$, we have

$$
\mathrm{d}_{\mathrm{n}-1}\left(\mathrm{e}_{2}\right)=\delta_{1}\left[\mathrm{e}_{\mathrm{n}}, \mathrm{e}_{0}\right]+\delta_{0}\left[\mathrm{e}_{1}, \mathrm{e}_{\mathrm{n}}\right] \Rightarrow 0=0
$$

Thus,

$$
\mathrm{d}_{\mathrm{n}-1}\left(\sum_{i=0}^{n} \lambda_{\mathrm{i}} \mathrm{e}_{\mathrm{i}}\right)=\lambda_{0} \delta_{0} \mathrm{e}_{\mathrm{n}}+\lambda_{1} \delta_{1} \mathrm{e}_{\mathrm{n}}
$$

This proves the proposition. we note these mappings are derivations and are linearly independent.

Corollary 2.1.

$$
\operatorname{dim} \operatorname{Der}\left(L_{n}\right)=\operatorname{dim} Z^{1}\left(L_{n}, L_{n}\right)=\mathrm{n}+2 .
$$

Corollary 2.2.

$$
\operatorname{dim} H^{1}\left(L_{n}, L_{n}\right)=n+1
$$

\section{Derivation of Filiform Leibniz Algebra $\mathbf{W}_{\mathbf{n}}$}

Proposition 3.1. The linear transformations $t_{1}, t_{2}, t_{3}, t_{4}, t_{5}$ and $\mathrm{d}_{\mathrm{k}}$ of $1 \leq k \leq n-2$ defined by the rules:

$t_{1}\left(\mathrm{e}_{0}\right)=\mathrm{e}_{0}, t_{1}\left(\mathrm{e}_{i}\right)=i \mathrm{e}_{i}, 2 \leq i \leq n$,

$t_{2}\left(\mathrm{e}_{0}\right)=\mathrm{e}_{1}$,

$t_{3}\left(\mathrm{e}_{1}\right)=\mathrm{e}_{1}$,

$t_{4}\left(\mathrm{e}_{0}\right)=\mathrm{e}_{\mathrm{n}}$,

$t_{5}\left(\mathrm{e}_{1}\right)=\mathrm{e}_{\mathrm{n}}$,

$d_{\mathrm{k}}\left(\mathrm{e}_{0}\right)=\mathrm{e}_{\mathrm{k}+1}, d_{\mathrm{k}}\left(\mathrm{e}_{i}\right)=\mathrm{e}_{\mathrm{k}+\mathrm{i}}, 2 \leq i \leq n-\mathrm{k}$.

form a basis of the space $\operatorname{Der}\left(\mathrm{W}_{\mathrm{n}}\right)$.

Proof. We introduce a grading of the algebra $W_{\mathrm{n}}=W_{1}$ $\oplus W_{2} \oplus \ldots \oplus$ by setting $W_{l}=\operatorname{lin}\left(\mathrm{e}_{0}, \mathrm{e}_{1}\right), W_{i}=\operatorname{lin}\left(e_{i}\right)$ for $2 \leq \mathrm{i} \leq$ $n$. Since $Z_{l}\left(W_{n}, W_{n}\right)=\operatorname{Der}(W n)$ and grading of the algebra $\mathrm{W}_{\mathrm{n}}$ is finite, there is a finite grading in the space $\operatorname{der}\left(\mathrm{W}_{\mathrm{n}}\right)$. Let $\mathrm{d}$ in $\operatorname{Der}(\mathrm{Wn})$. In this case, by lemma 1.1 we have $d=d_{0}+d_{1}+\ldots+d_{n}-2+d_{n-1}$ where $d_{i} \in \operatorname{Der}\left(W_{\mathrm{n}}\right)$ and $d_{i}\left(W_{j}\right) \subseteq W_{i+j}$.

Consider the element $\mathrm{d}_{0}$ in $\operatorname{Der}(\mathrm{Wn})$. It is clear that

$$
\mathrm{d}_{0}\left(\mathrm{e}_{i}\right)=\left\{\begin{array}{l}
\alpha_{\mathrm{o}} \mathrm{e}_{\mathrm{o}}+\alpha_{1} \mathrm{e}_{1}, \text { for } i=0, \\
\beta_{0}+\beta_{1} \beta_{1}, \text { for } i=1, \\
\gamma_{i} \mathrm{e}_{i,} \text { for } 2 \leq \mathrm{i} \leq \mathrm{n},
\end{array}\right.
$$

where $\alpha_{0}, \alpha_{1}, \beta_{0}, \beta_{1}, \gamma_{i}, 2 \leq i \leq n$, are scalars (elements of the field).

Consider the family of derivations

$$
\mathrm{d}_{0}\left(\left[\mathrm{e}_{i}, \mathrm{e}_{j}\right]\right)=\left[\mathrm{d}_{0}(\mathrm{e} i), \mathrm{e} j\right]+\left[\mathrm{e}_{\mathrm{i}}, \mathrm{d}_{0}\left(\mathrm{e}_{j}\right)\right]
$$

If $\mathbf{j}=1$ we obtain

$\left[\mathrm{e}_{i}, \mathrm{~d}_{0}\left(\mathrm{e}_{1}\right)\right]=\left[\mathrm{e}_{i}, \beta_{0} \mathrm{e}_{0}+\beta_{1} \mathrm{e}_{1}\right]=\beta_{0} \mathrm{e}_{i+1}=0 \Rightarrow \beta_{0}=0$.

If $\mathrm{i}=0$ and $\mathrm{j}=0$ then

$$
\mathrm{d}_{0}\left(\mathrm{e}_{2}\right)=\left[\mathrm{d}_{0}\left(\mathrm{e}_{0}\right), \mathrm{e}_{0}\right]+\left[\mathrm{e}_{0}, \mathrm{~d}_{0}\left(\mathrm{e}_{0}\right)\right] \Rightarrow \gamma_{2} \mathrm{e}_{2}=2 \alpha_{0} \mathrm{e}_{2}
$$

i.e., $\gamma_{2}=2 \alpha_{0}$

If $\mathrm{j}=0$ and $2 \leq i \leq n$ we obtain

$$
\mathrm{d}_{0}\left(\mathrm{e}_{\mathrm{i}+1}\right)=\gamma_{\mathrm{i}} \mathrm{e}_{\mathrm{i}+1}+\alpha_{0} \mathrm{e}_{\mathrm{i}+1} \Rightarrow \gamma_{\mathrm{i}+1}=\gamma_{i}+\alpha_{0} .
$$

i.e., $\gamma_{i=i} \alpha_{0}$

Thus,

$\mathrm{d}_{0}\left(\Sigma_{i=0}^{n} \lambda_{i} \mathrm{e}_{i}\right)=\lambda_{0}\left(\alpha_{0} \mathrm{e}_{0}+\alpha_{1} \mathrm{e}_{1}\right)+\lambda_{1} \beta_{1} \mathrm{e}_{1}+\alpha_{0} \Sigma_{i=2}^{n} \lambda_{i} \mathrm{e}_{\mathrm{i}}=\alpha_{0}$ $\left(\lambda_{0} \mathrm{e}_{0}+\Sigma_{i=2}^{n} \lambda_{i} \mathrm{e}_{\mathrm{i}}\right)+\alpha_{1} \lambda_{0} \mathrm{e}_{1}+\beta_{1} \lambda_{1} \mathrm{e}_{1}$

i.e., $\mathrm{d}_{0=} \alpha_{0} t_{1+} \alpha_{1} t_{2} \beta_{1} t_{3}$

Consider the elements $\mathrm{dk}$ in $\operatorname{Der}(\mathrm{Wn})$ for $1 \leq \mathrm{k} \leq \mathrm{n}-2$. It is clear that

$$
d_{\mathrm{k}}\left(\mathrm{e}_{i}\right)=\left\{\begin{array}{c}
\tau_{o} \mathrm{e}_{\mathrm{k}+1}, \quad \text { for } i=0, \\
\tau_{i} \mathrm{e}_{\mathrm{k}+\mathrm{i}}, \quad \text { for } 1 \leq \mathrm{i} \leq n-\mathrm{k},
\end{array}\right.
$$

where $\tau_{i}, 0 \leq i \leq n-\mathrm{k}$ are scalars (elements of the field).

Consider the following property of the derivations:

$$
d_{\mathrm{k}}\left(\left[\mathrm{e}_{i}, \mathrm{e}_{j}\right]\right)=\left[d_{\mathrm{k}}(\mathrm{e} i), \mathrm{e} j\right]+\left[\mathrm{e}_{\mathrm{i}}, d_{\mathrm{k}}\left(\mathrm{e}_{j}\right)\right]
$$

If $\mathrm{j}=0$ and $\mathrm{i}=1$, we have

$$
\tau_{1}\left[\mathrm{e}_{\mathrm{k}+1}, \mathrm{e}_{0}\right]=0 \Rightarrow \tau_{1} \mathrm{e}_{\mathrm{k}+2}=0 \Rightarrow \tau_{1}=0 .
$$

If $\mathrm{j}=0$ and $\mathrm{i}=0$, we have

$$
d_{\mathrm{k}}\left(\mathrm{e}_{2}\right)=\tau_{0}\left[\mathrm{e}_{\mathrm{k}+1}, \mathrm{e}_{0}\right] \Rightarrow \tau_{2} \mathrm{e}_{\mathrm{k}+2}=\tau_{0} \mathrm{e}_{\mathrm{k}+2} \Rightarrow \tau_{2}=\tau_{0} .
$$

if $\mathrm{j}=0$ and $2 \leq i \leq n-k$, we obtain

$$
d_{\mathrm{k}}\left(\mathrm{e}_{i+1}\right)=\tau_{i} \mathrm{e}_{\mathrm{k}+i+1}, \Rightarrow \tau_{\mathrm{i}+1} \mathrm{e}_{\mathrm{k}+\mathrm{i}+1}=\tau_{\mathrm{i}} \mathrm{e}_{\mathrm{k}+\mathrm{i}+1} \Rightarrow \tau_{\mathrm{i}+1}=\tau_{\mathrm{i}} .
$$

i.e., $\tau_{0}=\tau_{2}=\ldots=\tau_{\mathrm{n}-\mathrm{k}}$.

Thus,

$$
d_{\mathrm{k}}\left(\sum_{i=0}^{n} \lambda_{i} \mathrm{e}_{i}\right)=\tau_{0}\left(\lambda_{0} \mathrm{e}_{\mathrm{k}+1}+\Sigma_{i=2}^{n-k} \lambda_{i} \mathrm{e}_{\mathrm{k}+\mathrm{i}}\right) .
$$

Consider the elements $\mathrm{d} n-1 \in \operatorname{Der}\left(W_{n}\right)$. It is clear that 


$$
\mathrm{d}_{\mathrm{n}-1}\left(\mathrm{e}_{i}\right)= \begin{cases}\delta_{\mathrm{o}} e_{n}, & \text { for } \mathrm{i}=0, \\ \delta_{1} \mathrm{e}_{\mathrm{n}}, & \text { for } \mathrm{i}=1,\end{cases}
$$

where $\delta_{0}$, and $\delta_{0}$ are scalars (elements of the field).

Consider the following property of the derivations

$$
d_{n-1}\left(\left[\mathrm{e}_{i}, \mathrm{e}_{j}\right]\right)=\left[\mathrm{d}_{n-1}(\mathrm{e} i), \mathrm{e} j\right]+\left[\mathrm{e}_{\mathrm{i}}, d_{n-1}\left(\mathrm{e}_{j}\right)\right]
$$

If $\mathrm{i}=0$ and $\mathrm{j}=0$, we have

$$
d_{\mathrm{n}-1}\left(\mathrm{e}_{2}\right)=\delta_{0}\left[\mathrm{e}_{\mathrm{n}}, \mathrm{e}_{0}\right]+\delta_{0}\left[\mathrm{e}_{0}, \mathrm{e}_{\mathrm{n}}\right] \Rightarrow 0=0
$$

If $\mathrm{i}=1$ and $\mathrm{j}=0$, we have

$$
\delta_{1}\left[\mathrm{e}_{\mathrm{n}}, \mathrm{e}_{0}\right]+\delta_{0}\left[\mathrm{e}_{1}, \mathrm{e}_{\mathrm{n}}\right]=0 \Rightarrow 0=0
$$

Thus,

$$
d_{\mathrm{n}-1}\left(\sum_{i=0}^{\stackrel{n}{=}} \lambda_{\mathrm{i}} \mathrm{e}_{\mathrm{i}}\right)=\lambda_{0} \delta_{0} \mathrm{e}_{\mathrm{n}}+\lambda_{1} \delta_{1} \mathrm{e}_{\mathrm{n}} .
$$

i.e, $d_{\mathrm{n}-1}=\delta_{0} \mathrm{t}_{4}+\delta_{1} \mathrm{t}_{5}$ This proves the proposition. We note these mappings are derivations and are linearly independent.

Corollary 3.1.

$$
\operatorname{dim} \operatorname{Der}\left(W_{\mathrm{n}}\right)=\operatorname{dim} \mathrm{Z}_{1}\left(W_{\mathrm{n}}, W_{\mathrm{n}}\right)=\mathrm{n}+3 .
$$

Corollary 3.2.

$$
\operatorname{dim} H^{1}\left(W_{\mathrm{n}}, W_{\mathrm{n}}\right)=n+2
$$

\section{Conclusion}

Notations of this paper about any $(n+1)$ dimensions of some filiform Leibniz algebras $\left(\mathrm{L}_{\mathrm{n}}\right)$ and $\left(\mathrm{W}_{\mathrm{n}}\right)$.

1. notations about Derivations filiform Leibniz algebra $\left(\mathrm{L}_{\mathrm{n}}\right)$.

(a) The linear transformations $t_{1}, t_{2}, t_{3}, t_{4}$, and dk; $1 \leq k \leq n$ 2 of $\mathrm{Ln}$ are a basis of the space Der(Ln).

(b) We can find dimensions derivations of filiform Leibniz algebra (Ln), by using

$$
\operatorname{dim} \operatorname{Der}\left(L_{\mathrm{n}}\right)=\mathrm{n}+2 .
$$

(c) we can determine that the number equations of derivations for filiform Leibniz algebra Ln for any ( $\mathrm{n}+$ 1) dimensions such that $\mathrm{n} \geq 2$ by using

$$
\text { number Der }\left(L_{n}\right)=\left(n^{2}+5 n+2\right) / 2
$$

2. notations about Derivations filiform Leibniz algebra $\left(\mathrm{W}_{\mathrm{n}}\right)$.

(a) The linear transformations $t_{1}, t_{2}, t_{3}, t_{4}, t_{5}$ and $\mathrm{d} \mathrm{k}, 1 \leq k \leq$ $n-2$, of $\mathrm{Wn}$ are a basis of the space $\operatorname{Der}(\mathrm{Wn})$.

(b) We can find dimensions derivations of filiform Leibniz algebra $\left(\mathrm{W}_{\mathrm{n}}\right)$, by using

$$
\operatorname{dim} \operatorname{Der}(\mathrm{W} n)=\mathrm{n}+3 \text {. }
$$

(c) we con determine that number equations of derivations for filiform Leibniz algebra Wn for any $(n+1)$ dimensions such that $n \geq 2$, by using

numberDer $(W n)= \begin{cases}3 \mathrm{n}, & \text { when } \mathrm{n} \text { is even, } \\ \left(n^{2}+n+6\right) / 2, & \text { when } \mathrm{n} \text { is odd, }\end{cases}$

\section{Acknowledgements}

The research was supported by RUGS UPM (2009-2010).

\section{References}

[1] S. Albeverio, et al. , "n-Dimensional filiform Leibniz algebras of length (n-1) and their derivations," , Journal of Algebra, (2008), 319: 2471-2488.

[2] Sh. A. Ayupov and B. A. Omirov, "On some classes of nilpotent Leibniz algebras," , Sibirsk. Mat. Zh. [Siberian Math. J.], 42 (2001), no. 1, 18-29.

[3] N. Bourbaki, "Groupes et Algebras de Lie, Chap. I, Algebras de Lie," , Paris, 1960.

[4] C. Chevalley, "Theorie des Groupes de Lie, Tome II, Groupes algebriques,", Paris, 1951.

[5] J. Dixmier, "Sous-algebras de Cartan et decompositions de levi dans les algebrasde Lie," ,Trans. Roy. Soc. Canada Ser. III, 20 (1956), 17-21.

[6] J. Dixmier and W. G. Lister, "Derivations of nilpotent Lie algebras," , Proc. Amer. Math. Soc., 8 (1957), 155-158.

[7] M. Goze and Yu. Hakimdjanov , "Nilpotent Lie Algebras," , Mathematics and its Applications, vol. 361, Kluwer, Dordrecht, 1996.

[8] You. B. Hakimjanov, "variete des lois d'algebres de lie nilpotentes," , Geometrie Dedicata, 40 (1991), no. 3, 269-295.

[9] Harish-Chandra , "On the radical of a Lie algebra," , Prov. Amer. Math. Soc., 1 (1950), 14-17.

[10] J. E. Humphreys , "Introduction to Lie Algebras and Representation Theory," , Springer-Verlag New York. Heidelberg. Berlin. (1972), 25-27.

[11] N. Jacobson, A note on automorphisms and derivations of Lie algebras, Proc. Amer. Math. Soc. 6(1955), 281-283.

[12] Yu. B. Khakimdzhanov, "Characteristically nilpotent Lie algebras," , Mat. Sb. [Math. USSR-Sb.], 181 (1990), no. 5, 642-655.

[13] G. Leger, "A note on the derivations of Lie algebras," , Proc. Amer. Math. Soc., 4 (1953), 511-514.

[14] G. Leger , "Derivations of Lie algebras III," , Duke Math.J., 30 (1963), 637-645.

[15] J.- L. Loday and T. Pirashvili , "Universal enveloping algebra of Leibniz algebras and (co)homology,", Math. Ann., 296 (1993), no. 1,139-158.

[16] A. I. Malcev , "Solvable Lie algebras," , Izv. Akad. Nauk SSSR Ser. Mat., 9 (1945), 329-352.

[17] B. A. Omirov, "On the Derivations of Filiform Leibniz Algebras," , Mathematical Notes, Vol. 77, No5, 2005, 677-685.

[18] I.S. Rakhimov, and S.K. Husain, , "On isomorphism classes and invariants of low- dimensional Complex liform Leibniz algebras (Part 2)." , arXiv math RA. (2008). 
[19] E. Schenkman, "On the derivation algebra and the holomorph of a nilpotent Lie algebras," , Mem. Amer. Math. Soc. No. 14 (1955), 15-22.
[20] David J.Winter, Abstract Lie algebras, printed in the United States of America, (1972), $150 \mathrm{p}$. 